Network Working Group

Request for Comments: 2124

Category: Informational
P. Amsden

J. Amweg

P. Calato

S. Bensley

G. Lyons

Cabletron Systems Inc.

March 1997

\title{
Cabletron's Light-weight Flow Admission Protocol Specification Version 1.0
}

Status of this Memo

This memo provides information for the Internet community. This memo does not specify an Internet standard of any kind. Distribution of this memo is unlimited.

Abstract

Light-weight Flow Admission Protocol, LFAP, allows an external Flow Admission Service (FAS) to manage flow admission at the switch, allowing flexible Flow Admission Services to be deployed by a vendor or customer without changes to, or undue burden on, the switch.

Specifically, this document specifies the protocol between the switch Connection Control Entity (CCE) and the external FAS. Using LFAP, a Flow Admission Service can: allow or disallow flows, define the parameters under which a given flow is to operate (operating policy) or, redirect the flow to an alternate destination. The FAS may also maintain details of current or historical flows for billing, capacity planning and other purposes.

Table of Contents

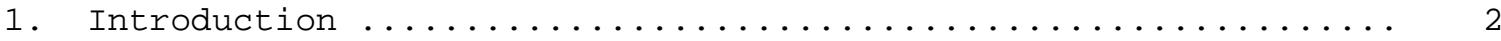

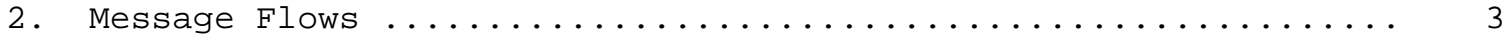

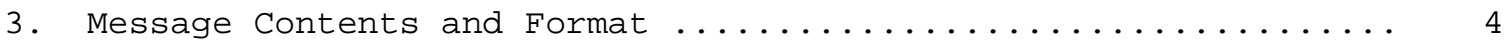

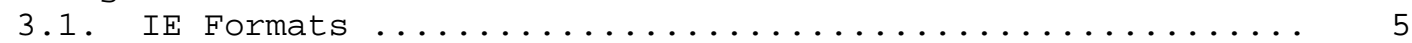

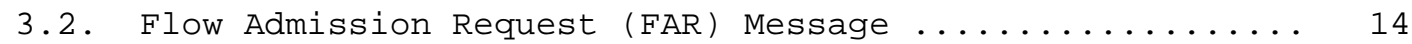

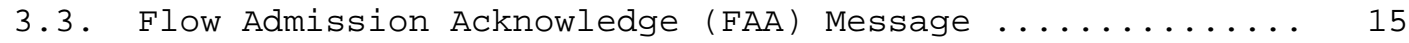

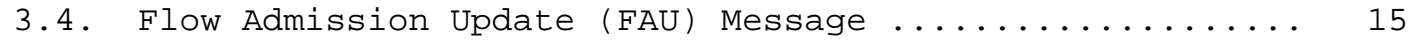

3.5 Flow Update Notification (FUN) Message .................. 16

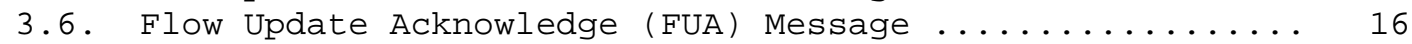

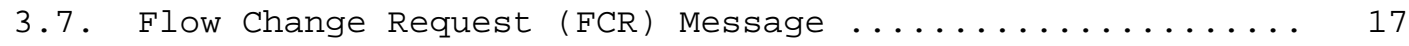

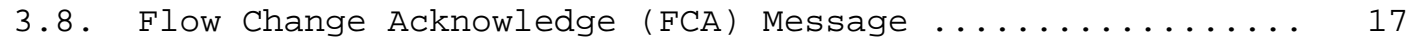

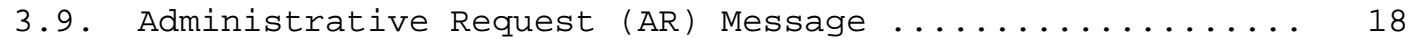

3.10. Administrative Request Acknowledge (ARA) Message ..... 18

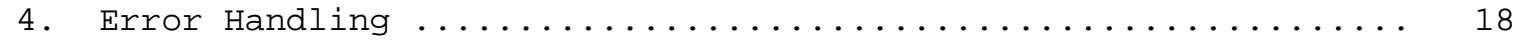




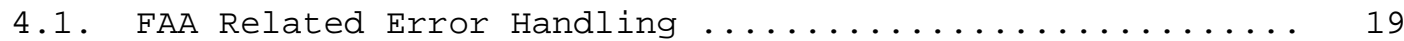

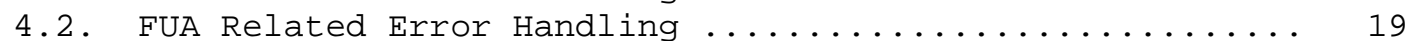

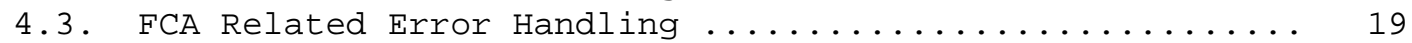

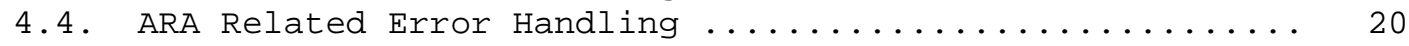

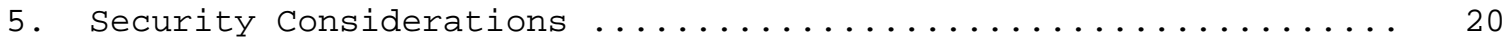

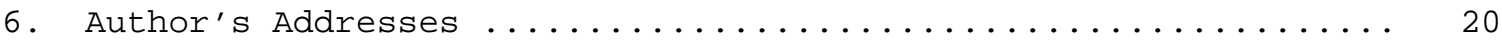

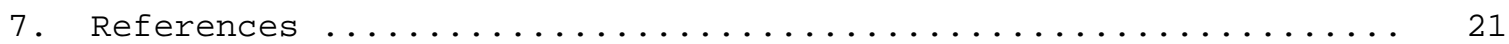

\section{Introduction}

Light-weight Flow Admission Protocol, LFAP, allows an external Flow Admission Service (FAS) to manage flow admission at the switch, allowing flexible Flow Admission Services to be deployed by a vendor or customer without changes to, or undue burden on, the switch. It provides a means for network managers, or management systems, to establish connection admission parameters for multiple switches in a single management domain by configuring policy information and other data via a single centralized connection admission control point.

Specifically, this document specifies the protocol between the switch Connection Control Entity (CCE) and the external FAS. Using LFAP, a Flow Admission Service can: allow or disallow flows, define the parameters under which a given flow is to operate (operating policy) or, redirect the flow to an alternate destination. The FAS may also maintain details of current or historical flows for billing, capacity planning and other purposes.

A significant advantage of this protocol is that it relieves switch vendors from the complexity of policy enforcement under any number of policy representation schemes. Similarly, switch configuration managers do not need to translate organization-determined policy or usage procedures, limitations and guidelines into an arbitrarily large set of vendor-specific representations. Finally, use of such a scheme makes possible plug-and-play connection management at the present time - in the absence of a standardized representation for connection policies.

This document describes the message flow between switch CCE and FAS, the messages used and error handling that applies. This constitutes the LFAP interface definition. 


\section{Message Flows}

Initiating message flows between $\mathrm{CCE}$ and FAS entities always originate at the switch. Therefore, the switch is the point at which connectivity is originated. The CCE must have IP reachability using some approach described elsewhere (e.g. [1577] or [LANE]) and an IP address for the FAS must be preconfigured at the switch CCE. The CCE establishes TCP connectivity using the registered port number - \#\#\#.

As shown below, Flow Admission Request (FAR) messages are sent by a switch's Call Control Entity (CCE) to the Flow Admission Service (FAS). These messages are sent when a flow is about to be set up by the switch and contain specific information relating to the flow such as flow identifier, source/destination and qualifying information about the flow - that may be required to determine the admissibility of the flow and any operating policies that apply to the flow if it is admitted.

The FAS responds with a Flow Admission Acknowledge (FAA) message (to the CCE) with a status indicating connection admissibility and any operating policy information that applies to the flow. If a FAA message contains mandatory operating policies that the switch CCE does not understand, the switch would abort the flow using the Flow Admission Update (FAU) message.

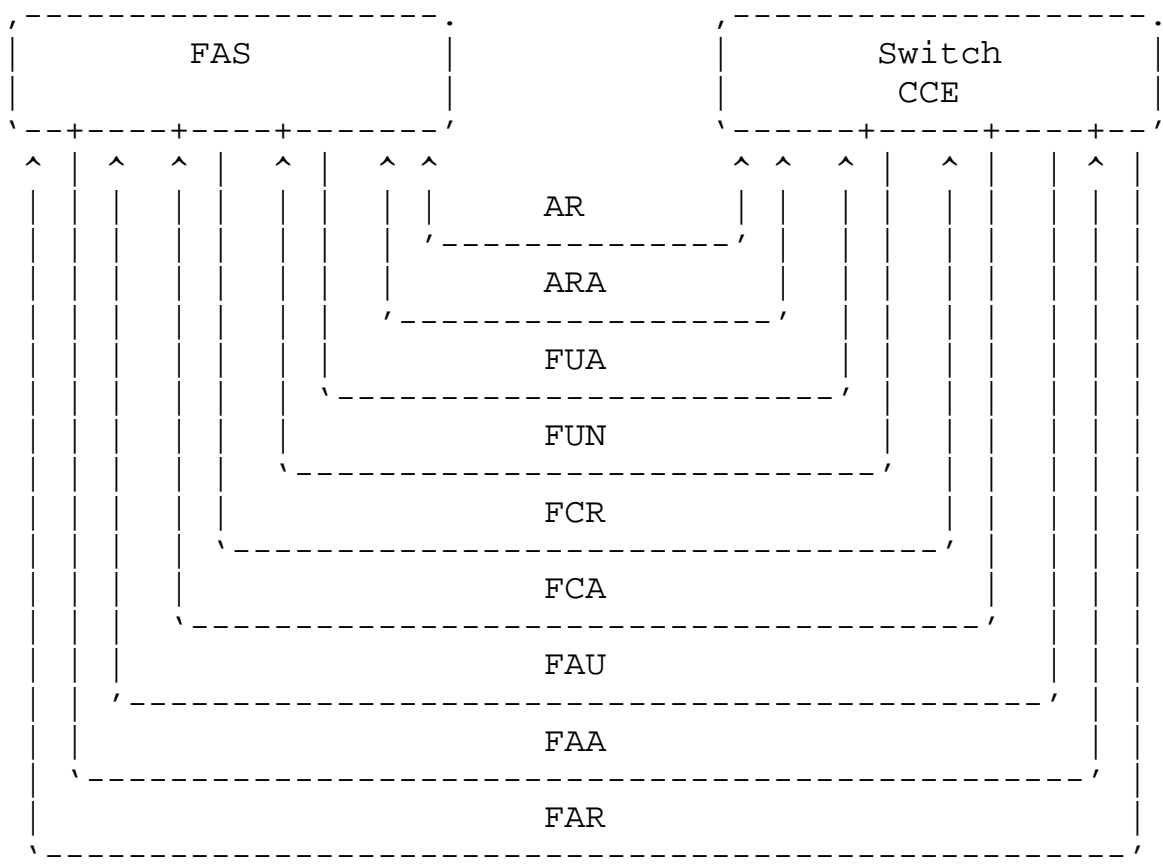


When a connection is established, periodically during the course of maintaining the connection and when a change in connection state occurs, the switch CCE sends a Flow Update Notification (FUN) message to the FAS. The FAS, in turn, responds with a Flow Update Acknowledge (FUA) message with a Flow failure code if a an error condition has been detected. An example of error conditions would be receipt of a FUN message indicating octets received and sent for a connection never admitted.

The FAS may send a Flow Change Request (FCR) to the CCE either to effect a change in the state of a specific connection or to set any new/changed policy information that applies to the flow.

The CCE replies with a Flow Change Acknowledge (FCA) message and may respond with a flow failure code indicating the offending flow or policy change.

Either the CCE or the FAS may initiate a Administrative Request (AR). The CCE uses it to get a Flow Identifier Prefix. The FAS uses it to request FUN messages be returned on some set of flows.

The requested entity (FAS or CCE) replies with a Administrative Request Acknowledge. The FAS uses the ARA to return the requested Flow Prefix. The CCE uses the ARA to return any Flow Identifiers that were in error on the AR.

3. Message Contents and Format

LFAP defines nine messages: "Flow Admission Request", "Flow Admission Acknowledge", "Flow Admission Update", "Flow Update Notification", "Flow Update Acknowledge", "Flow Change Request", "Flow Change Acknowledge", "Administrative Request" and "Administrative Request Acknowledge" (FAR, FAA, FAU, FUN, FUA, FCR, FCA, AR, ARA

respectively).

FAR messages are sent by a switch call control entity (CCE) to the Flow Admission Service (FAS). FAA messages are responses from the FAS to the CCE. FUA messages are responses from the CCE only under error conditions. FUN messages originate at switches and are acknowledged by FUA messages from the FAS. FCR messages are sent by the FAS to the $\mathrm{CCE}$ and are acknowledged by FCA messages. AR messages are sent by either the Entity (FAS or CCE) and are acknowledged by the ARA messages. 


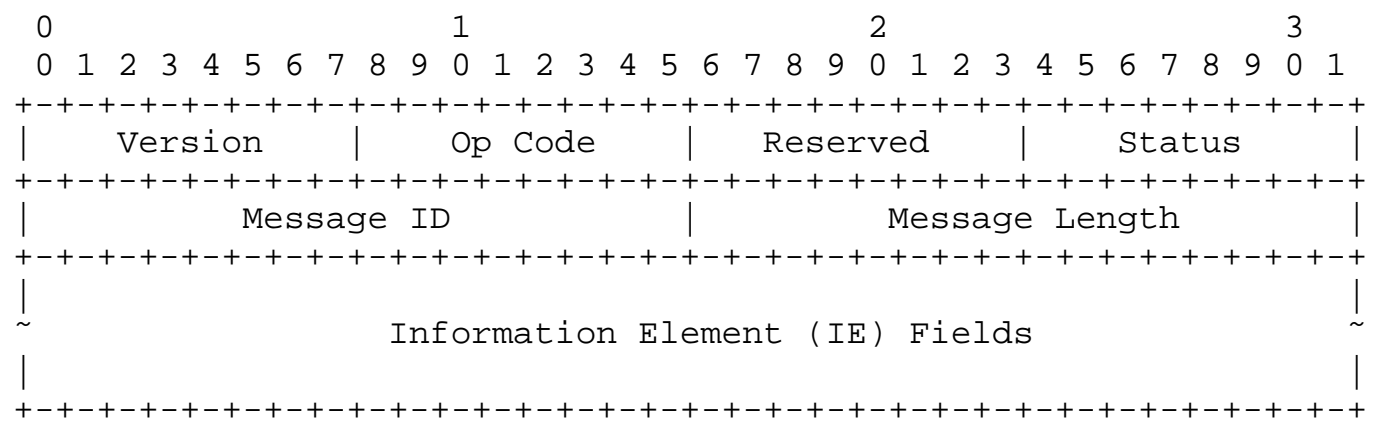

The general message format for all LFAP messages is as shown above. Version is 1 and Op Codes are as follows:
FAR - 1
FAA - 2
FAU - 3
FUN - 4
FUA - 5
FCR - 6
$\mathrm{FCA}-7$
$\mathrm{AR}-8$
ARA - 9

The Status field serves as a status on the overall message. The values that status may assume are:

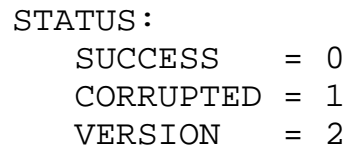

Message ID is used to associate each original message with its corresponding response and must be unique for the combination of sender and responder while an original message is pending. The Message Length excludes the 8 octets of the message header.

\subsection{IE Formats}

IE fields consist of 2-octet TYPE, 2-octet LENGTH and a variable length VALUE sub-fields. All IEs are even multiples of 4 octets in length, left-aligned and zero filled if necessary. Length is computed excluding the 4 octet TYPE and LENGTH fields.

Individual IEs are formated as described in following sections. 
Byte count IE

Contains the count of octets sent and received associated with the identified connection. IE format is:

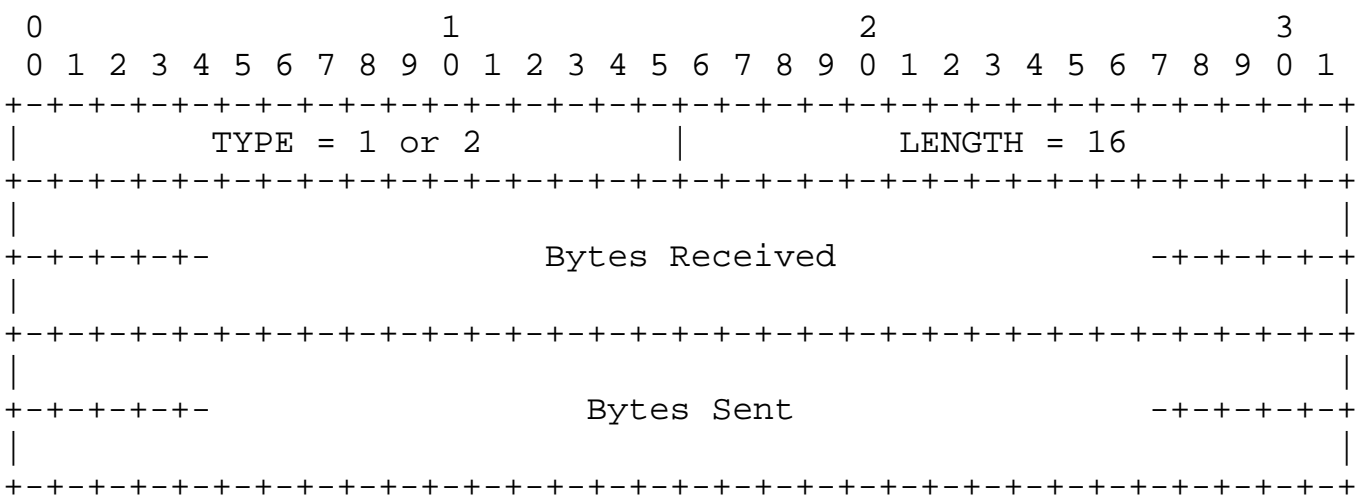

Type 1 Means that the byte count is a running counter and is the count from the beginning of the flow establishment.

Type 2 Means that the byte count is a delta counter and is the count since the last FUN message.

Packet count IE

Contains the count of packets cells or frames sent and received associated with the identified connection. IE format is:

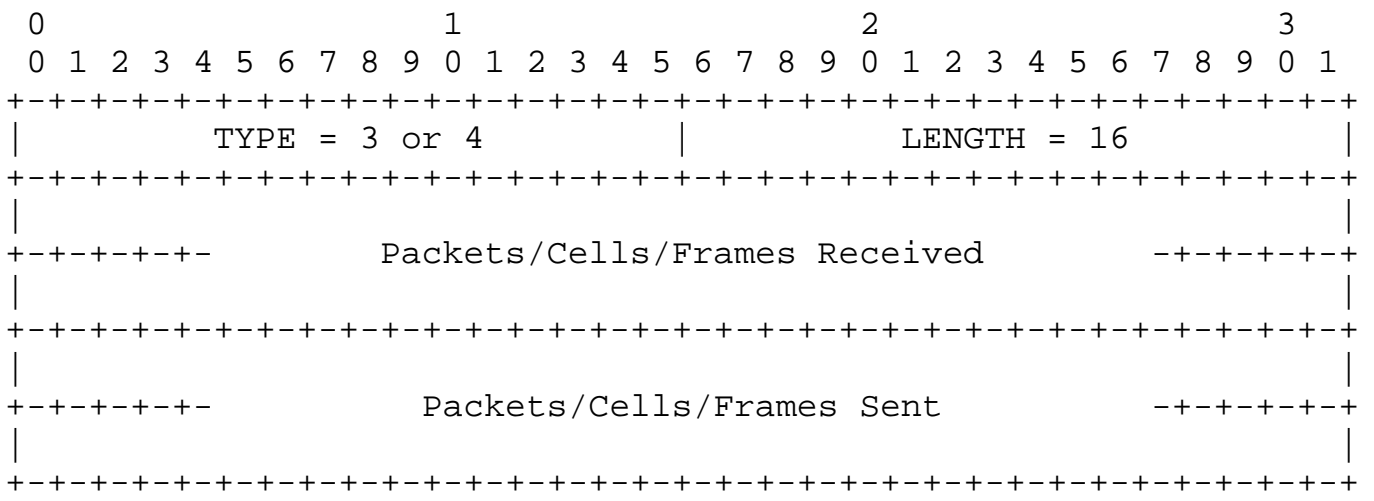

Type 3 Means that the packet/cell/frame count is a running counter and is the count from the beginning of the flow establishment.

Type 4 Means that the packet/cell/frame count is a delta counter and is the count since the last FUN message. 


\section{Client Data IE}

For use in determination of admission policy relative to a specific connection request based on arbitrary client data (OCTET STRING [8824]). IE format is:

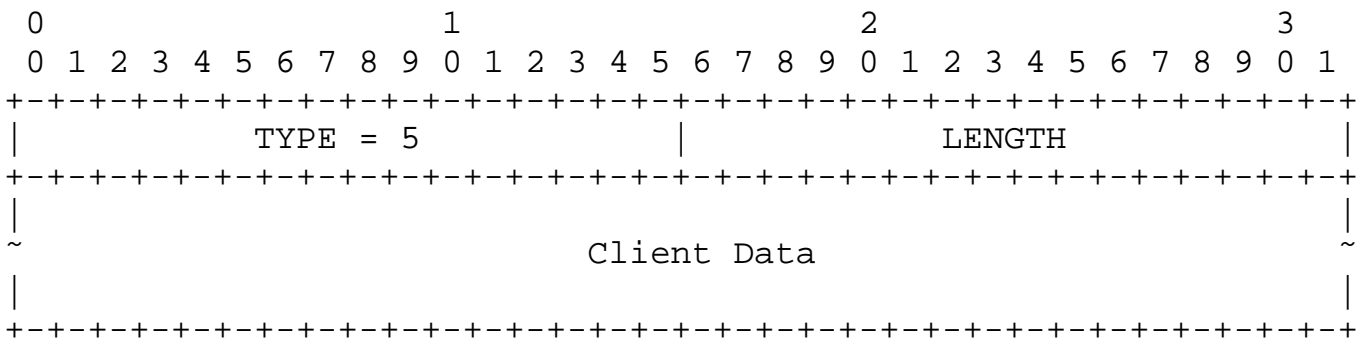

Destination Address IE

Destination address associated with a message. IE format is:

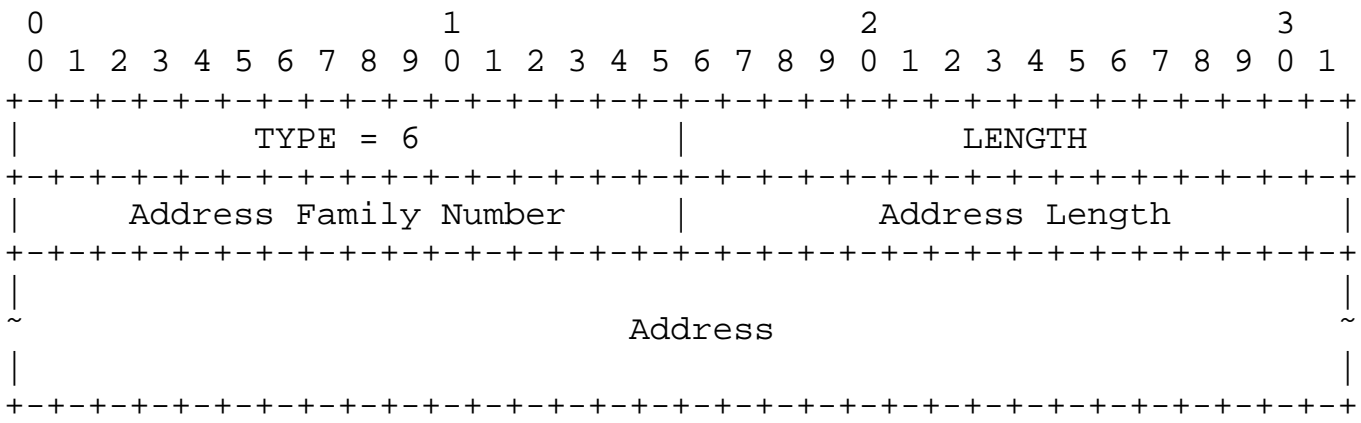

The Address Length field contains the length of the address excluding any pad of zeros used to align the address field.

Address Family Numbers include:

$$
\begin{aligned}
& 1-14 \text { (decimal) as specified in [1700] } \\
& 15 \\
& \text { E.164 with NSAP format subaddress }
\end{aligned}
$$

Flow ID IE

In order to accumulate the flow accounting statistics across multiple FAS's in case of a FAS failure a globally unique flow identifier needs to be formed. To accomplish this the FAS assigns a prefix if requested by the $\mathrm{CCE}$. The $\mathrm{CCE}$ then assigns a CCE flow identifier that it guaranties to be unique for the use of the FAS flow identifier prefix for each flow admitted. If the CCE needs to reuse 
a CCE flow ID it must acquire a new FAS prefix. If a CCE cannot support the FAS flow identifier then it does not request a FAS prefix and uses a FAS length of 0 in all updates to the flow. If the CCE does not support the FAS identifier prefix then when a CCE fails over all calls will need to be readmitted and will be seen as two separate calls at the accumulation point. Flow ID IE is copied exactly in all messages that refer to this flow. IE format is:

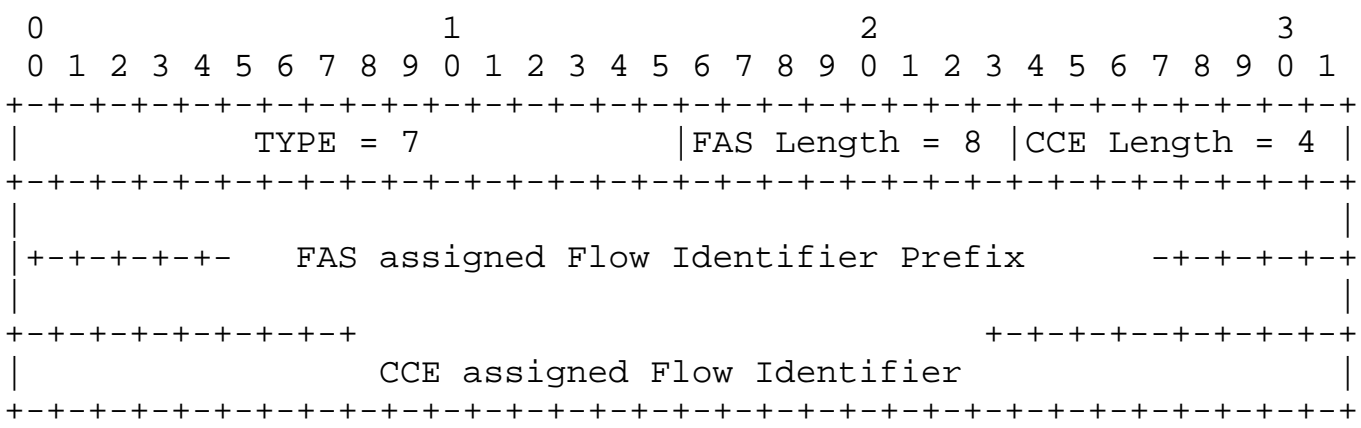

Flow State IE

Flow state is the intended end state for the Flow associated with the message containing this IE. IE format is:

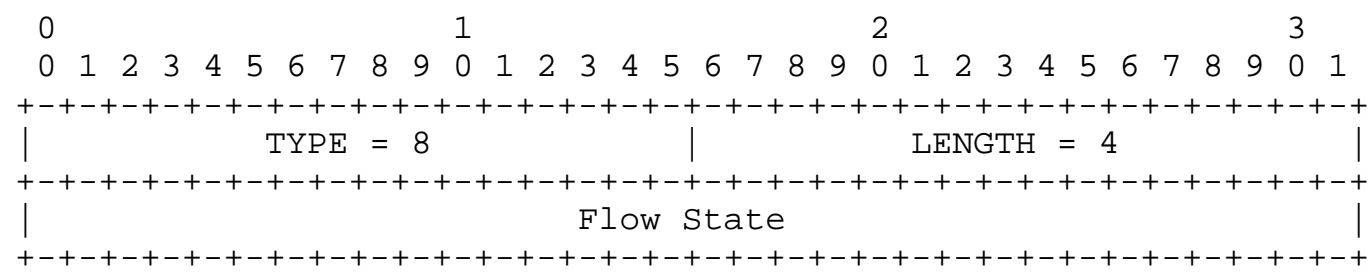

Flow state has one of the following values and meanings:

$$
\begin{array}{ll}
0-\text { INACTIVE } & - \text { Flow is inactive } \\
1-\text { ACTIVE } & - \text { Flow is active }
\end{array}
$$

Policy IE's

There are two basic types of Policy IE's Optional and Mandatory. In the case of optional operating policy if the combination of policy and value given cannot be interpreted by a switch CCE it may be safely ignored. In the case of mandatory operating policy if the combination of policy and value given cannot be interpreted by a switch CCE it must abort the flow state. Examples of optional operating policies are Checkpoint Timer and Connection Priority. 
There are also two forms of the policy ID. The first is where the policy ID is a number and the second is where the policy ID is an Object Identifier. The policy ID's with number values are identified in this document and its proposed changes over time. The object Identifier IDs can be used by individual implementers to apply proprietary or experimental additions to this document and still be compliant with the general form of this document.

Operating Policy IEs are comprised of Policy ID, a length and a value. In the case of the policies defined in this document a length is required and specified here. In the case of policies using the OID format the length may be implied by the OID or be part of the policy value as determined by individual implementation.

Policy IE format for Policy ID's defined in this document

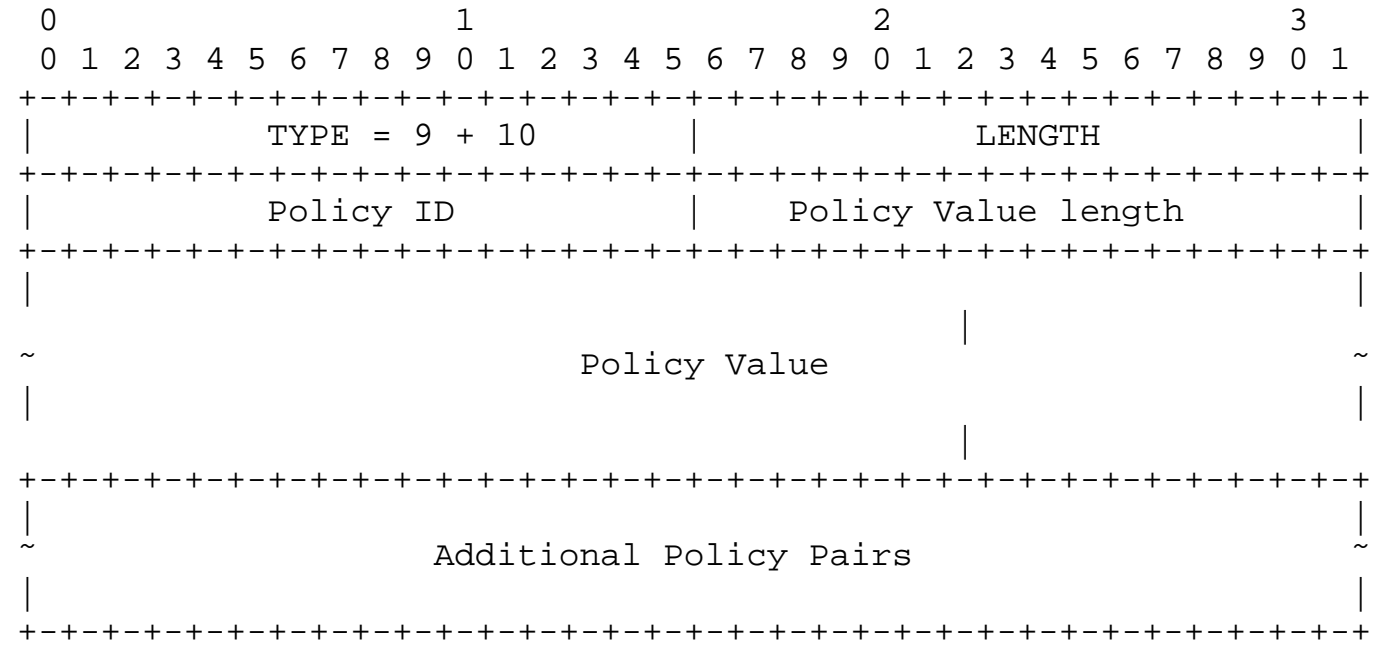

Type 9 is an Optional Policy and type 10 is a Mandatory Policy.

The following policy ID's and policy values are presently defined or under consideration.

Policy ID Value Meaning

Usage $\quad 01 x x \quad$ The purpose of this set of

policies is for usage constraints. This set of policies in the future may include Connection Count, Maximum Bandwidth and Connect Time. 


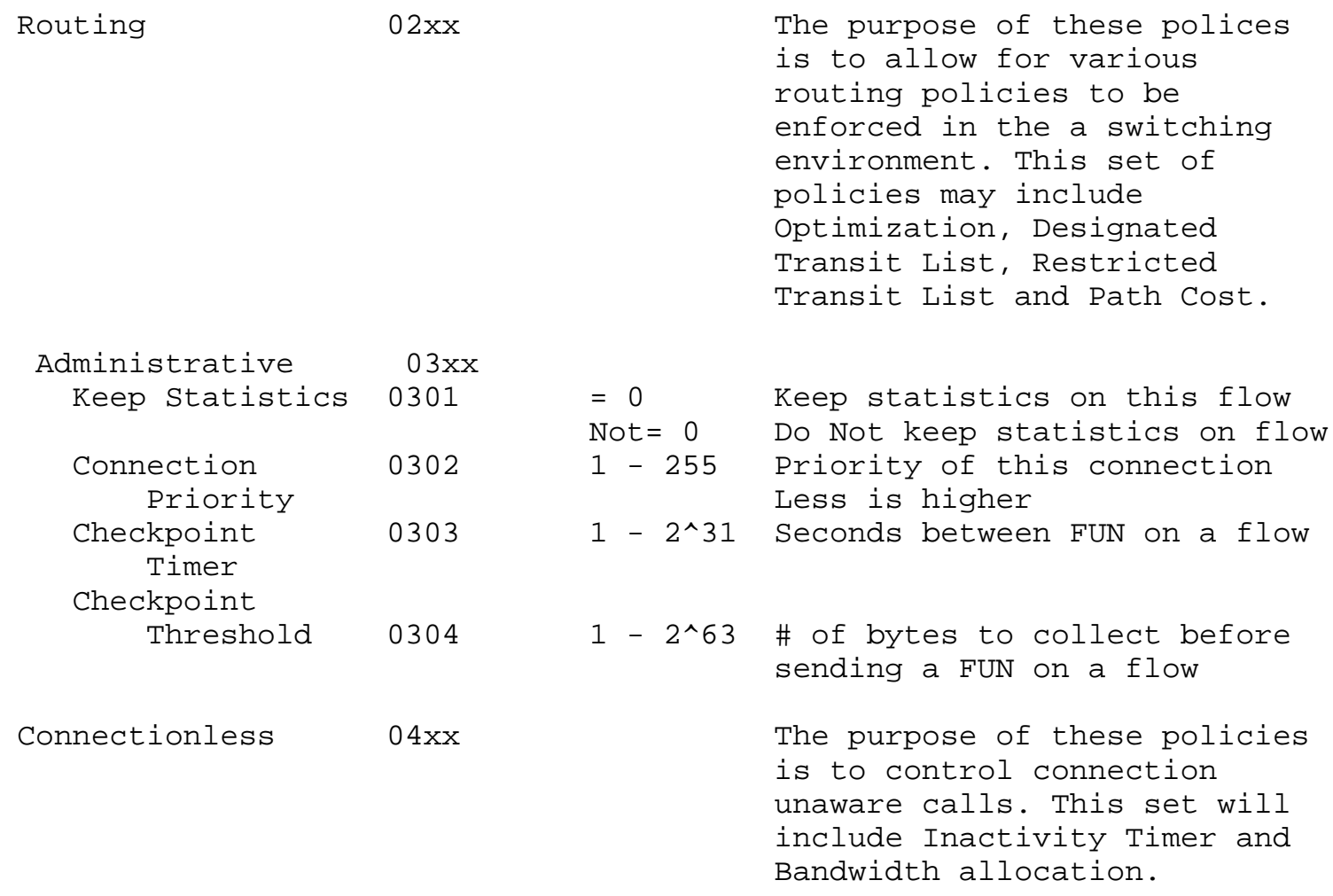

Policy IE format when Policy ID is a Object Identifier

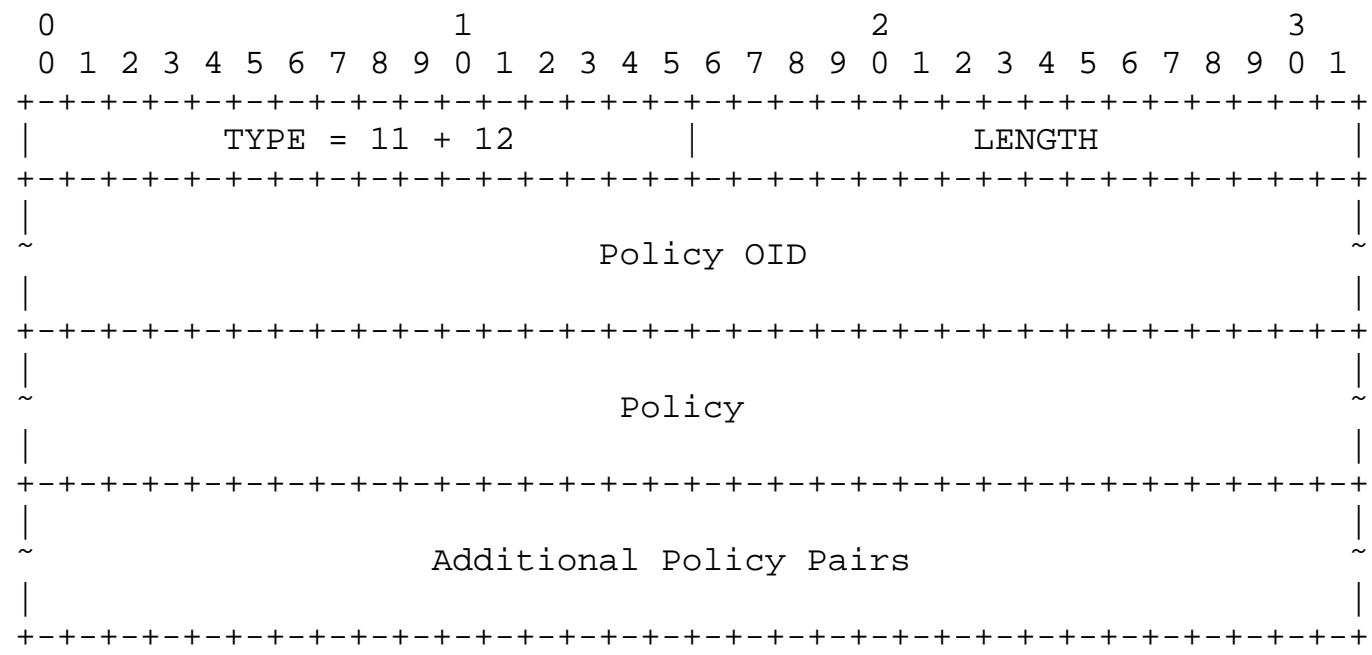

Type 11 is an optional policy and type 12 is a mandatory policy. 
Service Identifier IE

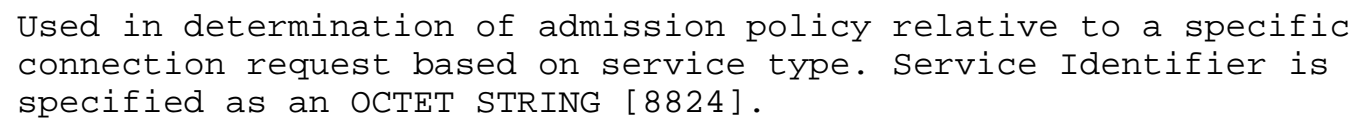

Source Address IE

Source address associated with a message. TYPE is 14, format is as shown for Destination Address IE.

Source Switch Address IE

Source Switch address associated with a message. TYPE is 15, format is as shown for Destination Address IE.

Destination Switch Address IE

Destination Switch address associated with a message. TYPE is 16 , format is as shown for Destination Address IE.

Time IE

The time (as a SNMPv2 Timestamp [1443]) associated with the status/statistics observed. TYPE is 17 and format is:

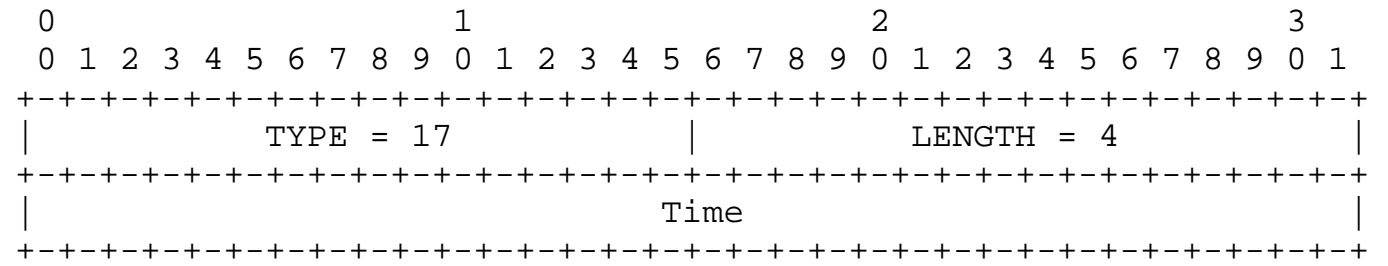


Multiple Record IE

The Multiple Record IE is composed of 4 parts. The record descriptor, fixed information, record format IEs and individual records. The record descriptor consist of two fields the first field is the length of the fixed information field. The second is the length of the Record format section. The fixed information is the IE's that apply to all the records that follow. The Record Format is the list of IE's that make up each record. The individual record section contains the individual records that are being reported in the format given by the Record Format section.

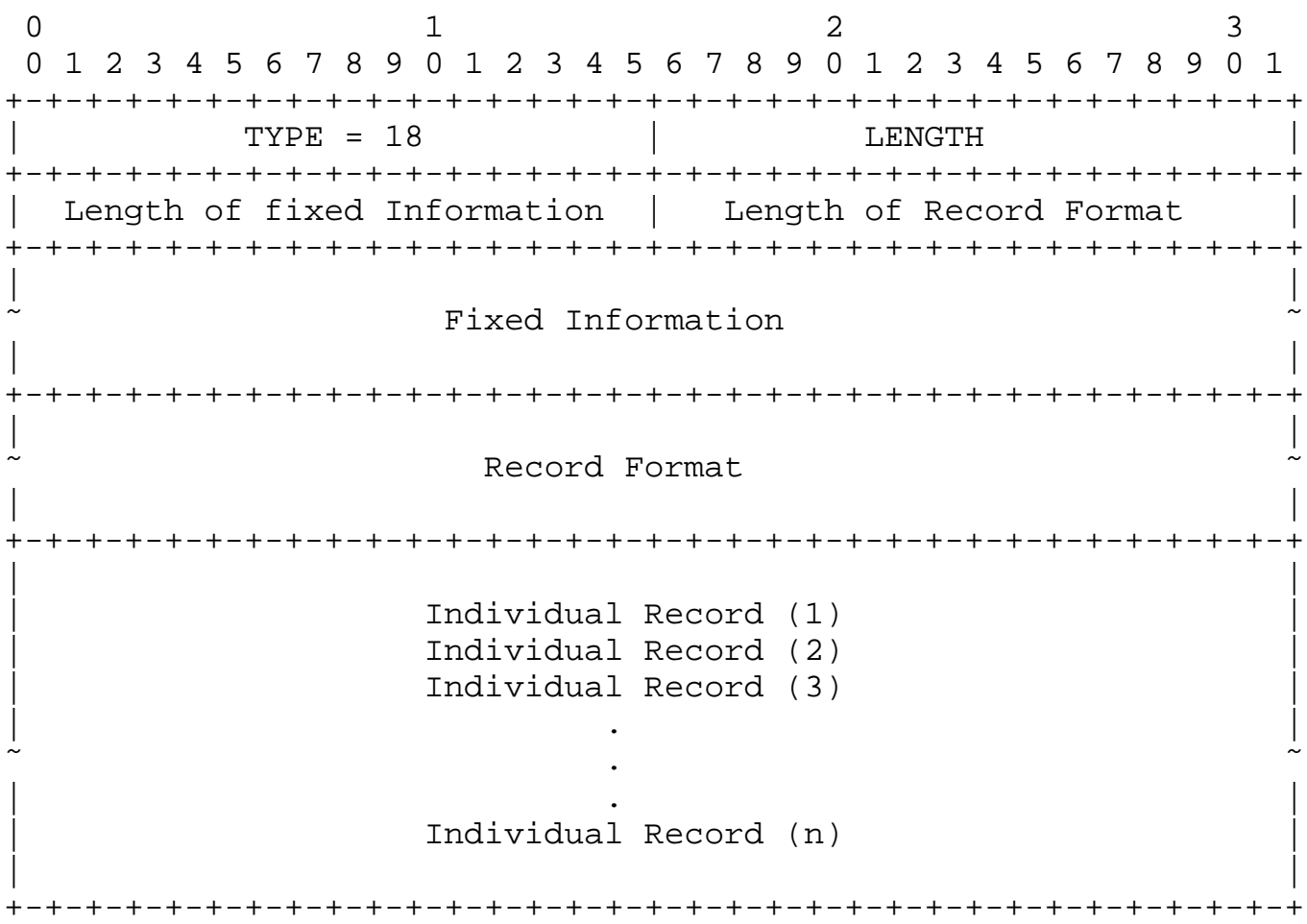


Flow Failure Code IE

Flow failure code is the reason why a operation an a given flow failed. IE format is:

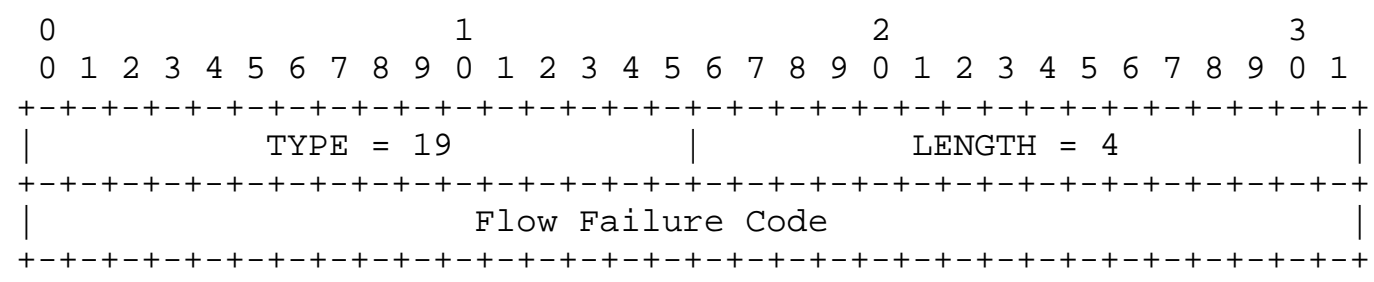

Flow failure code has one of the following values and meanings:
0 - POLICY_REJECT
- A policy reject has occurred
1 - NO_SUCH_FLOW
- The specified was flow was not found
2 - AMBIGUOUS
- Duplicate FAR caused this error
3 - DESTINATION_UNKNOWN
- The redirect destination was unknown

Command Code IE

Command Code is a administrative request command to perform a particular function. IE format is:

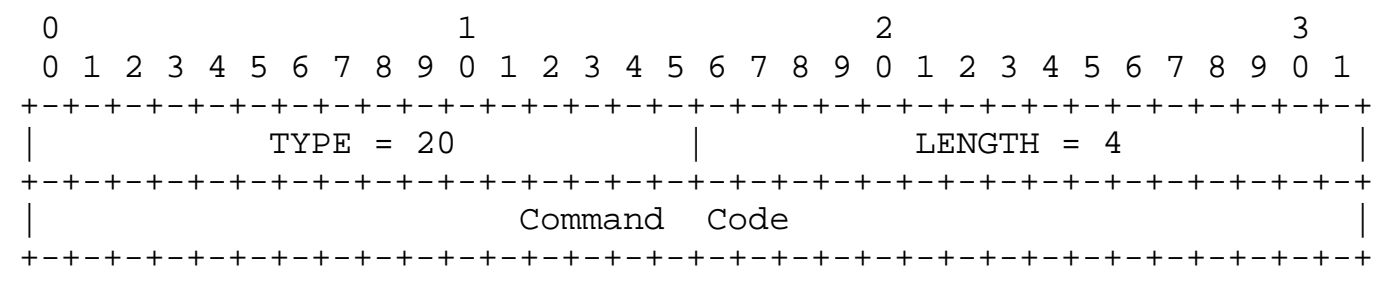

Command code has one of the following values and meanings:
0 - RETURN_INDICATED_FLOWS
- Return FUNs indicated
1 - RETURN_ALL_CHANGED_FLOWS
- Return FUNs indicated
2 - RETURN_ALL_FLOWS
- Return FUNs indicated
3 - RETURN_FLOW_PREFIX
- Return a new flow prefix 
Flow Identifier Prefix IE

The flow Identifier prefix IE gives the prefix that the FAS has created to maintain a globally unique ID. IE format is:

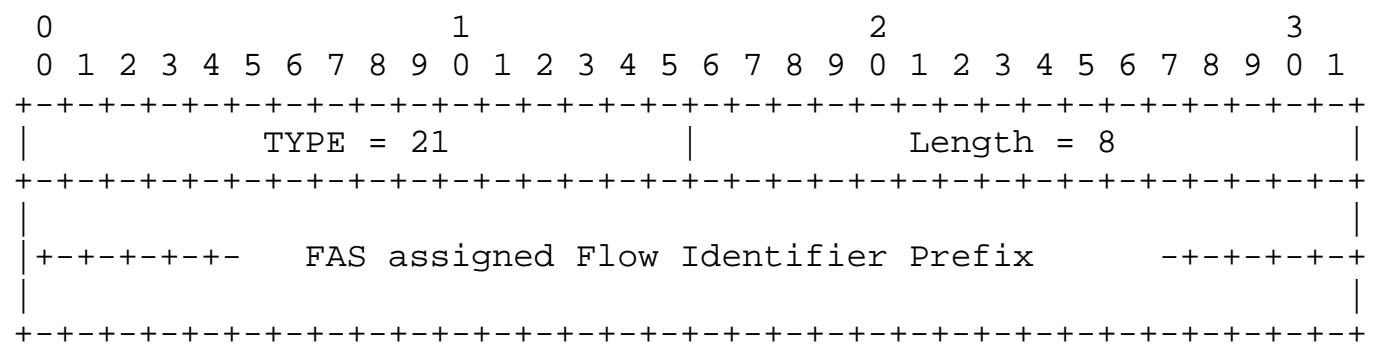

3.2. Flow Admission Request (FAR) Message

Status is set to SUCCESS in FAR messages. In addition, FAR messages may contain the following IEs:

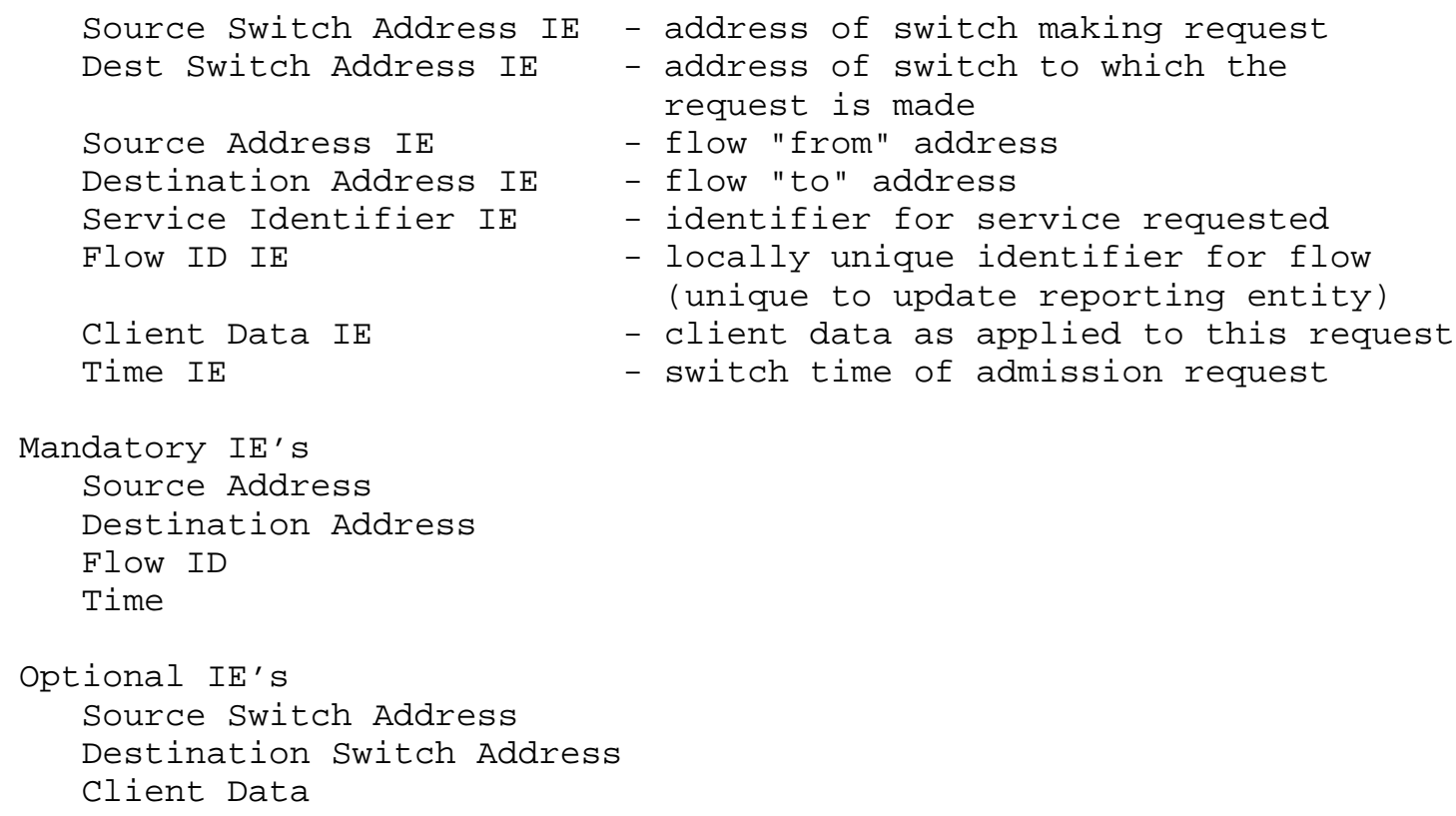


FAR messages are sent by a switch CCE when it seeks verification of validity of a flow that is about to be established. FAR messages refer to a single flow only and do not multi IE functionality. Source and destination addresses are those determined by the switch CCE as the points of origin and termination of a flow. Service ID is the service type/category associated with the desired flow. The client data is arbitrary information about the client associated with the desired flow.

\subsection{Flow Admission Acknowledge (FAA) Message}

Status reflects the result of the corresponding FAR message (see Error Handling for details). Message ID is copied from the FAR message. In addition, FAA messages may have the following IEs:

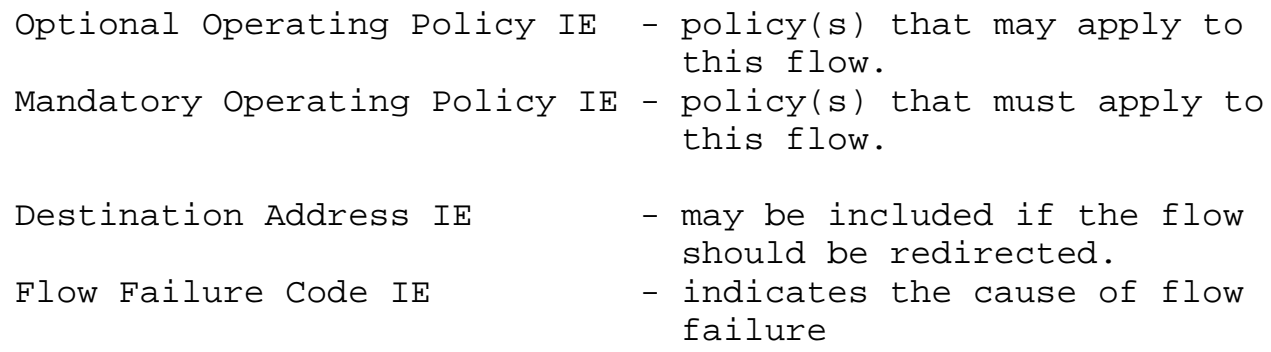

FAA messages are sent by a FAS in response to FAR messages received from a switch CCE. Operating policy information is that determined by the FAS as either desirable or required for the flow specified in the corresponding FAR message.

\subsection{Flow Admission Update (FAU) Message}

Status reflects the result of the corresponding FAA message (see Error Handling for details). Message ID is copied from the FAR or FAA message. In addition, FAU messages may have the following IEs:

Flow ID IE - identifier for the flow

Flow Failure Code IE - indicates the cause of flow failure

FUA messages are sent by a switch CCE in response to FAA messages received from a FAS. The FAU message is returned by the switch CCE only if an a error was detected as a result of the FAA message. 


\subsection{Flow Update Notification (FUN) Message}

Status is set to SUCCESS in FUN messages. In addition, FUN messages may contain the following IEs:

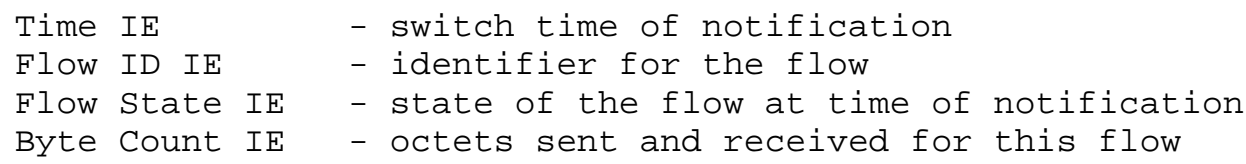

FUN messages are sent periodically (possibly as specified in an operating policy associated with the flow) by an CCE to the FAS. The Time IE may be given first and only once. If it is only a single flow being reported on then just the IE'S and their values are returned. If multiple flows are to be reported on then the multiple record IE should be used. This results in reduced overhead on transmissions. FUN messages may are also be sent as a result of a AR message or a FCR message. The FCR message would be one that request that the flow state be set to inactive.

The flow ID identifies the flow to which this update applies. Flow state is the state of the flow at the time this message is sent. Counts are as specified in the IE definitions. The FAS's are coordinated and will resolve the reception of FUN information from a CCE who has lost connection with its FAS and has gone to a alternative FAS.

3.6. Flow Update Acknowledge (FUA) Message

Status is set to SUCCESS in FUA messages unless an error is detected (see "Error Handling"). Message ID is copied from the FUN message. 
FUA messages are sent by the FAS to acknowledge a FUN message from the switch CCE. If a FUN message contained a multiple record IE and any of the updates had a error then the FUA would contain a multiple IE with a Flow ID and Flow Failure Code. A status of SUCCESS indicates that the information in the corresponding FUN message has been accepted and is now the responsibility of the FAS.

\subsection{Flow Change Request (FCR) Message}

Status is set to SUCCESS in FCR messages. In addition, a FCR message may contain the following IEs:

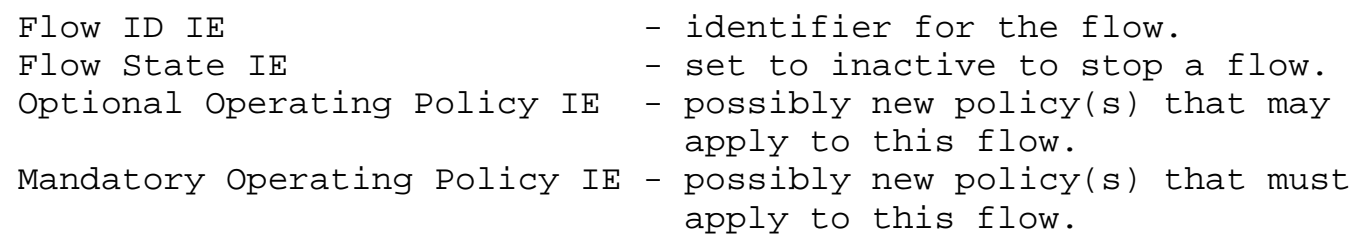

FCR messages are sent by the FAS to the CCE to provide additional (or change existing) operating policy information or to stop a flow. Flow ID is used to identify the flow to which this message applies. The FAS can stop a flow by setting it's flow state to inactive. This will cause the CCE to generate a FUN message with the final flow statistics. It will also cause the $\mathrm{CCE}$ to return a inactive flow state on the given flow. If the FAS wishes to change operating policy information it merely includes the new information in the FCR message along with the flow id.

\subsection{Flow Change Acknowledge (FCA) Message}

Status is set to SUCCESS in FCA messages unless an error is detected (see "Error Handling"). Message ID is copied from the FCR message. FCA messages contain IEs if a error was detected in the corresponding FCR message (see "Error Handling").

If an error occurs then a FCR may contain the following IE's

Flow ID IE - if FAS requested statistics on an unknown flow. 
Flow Failure Code - for the Flow ID IE above.

FCA messages are sent by a switch CCE in response to an FCR.

\subsection{Administrative Request (AR) Message}

Status is set to SUCCESS in AR messages. In addition, AR messages may contain the Command IEs:

Mandatory IE's

Command IE

AR messages are sent by either the a switch CCE or the FAS when they seeks to perform one of the Command $I E^{\prime} \mathrm{s}$.

\subsection{Administrative Request Acknowledge (ARA) Message}

Status reflects the result of the corresponding AR message (see Error Handling for details). Message ID is copied from the AR message. In addition, ARA messages may have the following IEs:

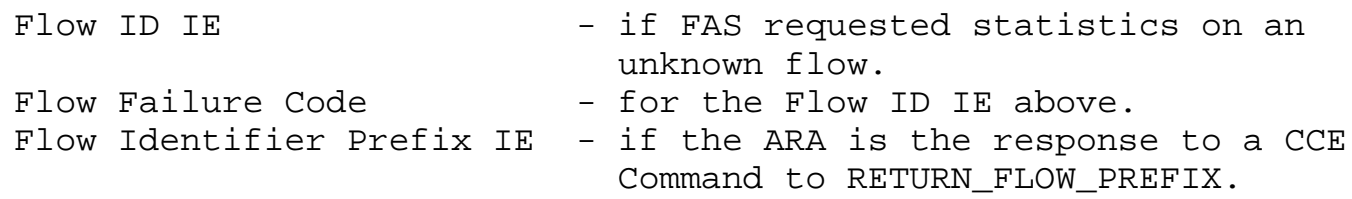

ARA messages are sent by a FAS or CCE in response to AR message received CCE or FAS respectively.

4. Error Handling

Incompatible version - Receipt of any LFAP request or notification message, with a version number other than that (or those) supported by the receiving component will result in a response (acknowledge) message with a status of VERSION. The resulting message will contain no IEs and, as a result, may be considered a generic FAILURE message.

Corrupted message contents - Receipt of a LFAP message which cannot be understood will result in a similar generic FAILURE message with Status set to CORRUPTED. A FAA message may contain a Flow ID IE only if this IE is included in the portion of the corrupt LFAP message that is before the point where corruption occurs. The LFAP sender should re-send the original message at least one time if it is still desired to admit the requested connection. 
With the exception of incompatible version and corrupted message contents, error handling is naturally related to the processing of response messages by both response sender and receiver. Below sections are thus organized around processing of FAA, FUA, FCA and ARA messages.

\subsection{FAA Related Error Handling}

Non-unique Flow ID - Receipt of a FAR message with a non-unique Flow ID may occur for two reasons: the CCE may have re-sent a FAR message and an error may have occurred in the ID generation function. If the entire message is the same in every respect, with the possible exception of Message ID, as a FAR message received previously, the FAS will respond in the same way as it would have responded to that prior message. Otherwise, the Flow ID will be returned with a Flow Failure Code set to AMBIGUOUS. The CCE should choose a new Flow ID and retry the FAR message if it is still desired to admit the requested connection.

Flow is inadmissible - The FAS may determine that flow is not admissible for policy reasons. In this case the Flow ID is returned along with the Flow Failure Code of POLICY_REJECT.

\subsection{FUA Related Error Handling}

Flow Not Admitted - Receipt of Flow information for an unadmitted connection. Flow ID IE identifies a flow which was not admitted or for which admission status has been lost. The FAS will return the Flow ID and a Flow Failure Code of NO_SUCH_FLOW. The switch CCE should send an appropriate FAR message. The FAS may track occurrences of this error and send a FCR message to the CCE requesting dropping of the reported connection.

\subsection{FCA Related Error Handling}

Flow change requested for a non-existent flow - The Flow ID IE identifies a connection for which this CCE has no state information. The FCA message has the Flow ID and a Flow Failure code set to NO_SUCH_FLOW and contains the Flow ID and copied from the corresponding FCR message.

Policy changes requested were not supported by the CCE. The FCA message has the Flow ID and a Flow Failure Code set to POLICY_REJECT and contains the Flow ID copied from the corresponding FCR message. 
4.4. ARA Related Error Handling

Flow statistics requested for a non-existent flow - The Flow ID IE identified a connection for which this CCE has no state information. The ARA message has the Flow ID and a Flow Failure code set to NO_SUCH_FLOW and contains the Flow ID copied from the corresponding FCR message. If there were multiple flows that were non-existent then the multi ie format could have the Flow Failure code in the fixed information section and the individual Flow ID's in the record section.

5. Security Considerations

Security issues are not discussed in this memo.

6. Author's Addresses

Paul Amsden

Phone: +1 (603) 337-7408

EMail: amsden@etron.com

Jim Amweg

Phone: +1 (603) 337-5247

EMail: amsdendetron.com

Paul Calato

Phone: +1 (603) 337-7625

EMail: amsden@etron.com

Stephen Bensley

Phone: +1 (603) 337-7061

EMail: amsden@etron.com

Gregory Lyons

Phone: +1 (603) 337-5318

EMail: amsden@ctron.com

Cabletron Systems, Inc. is located at:

P.O. Box 5005

Rochester, NH, 03866-5005

USA 
7. References

[363] "B-ISDN ATM Adaptation Layer (AAL) Specification," International Telecommunication Union, ITU-T Recommendation I.363, Mar. 1993.

[1443] "Textual Conventions for version 2 of the Simple Network Management Protocol (SNMPv2)", RFC 1443, April 1993.

[1700] Reynolds, J., and J. Postel, "Assigned Numbers", STD 2, RFC 1700, October 1994.

[8824] Information technology - Open Systems Interconnection "Specification of Abstract Syntax Notation One (ASN.1), Second edition", ISO/IEC TR 8824: 1990 (E) 1990-12-15.

[9577] "Telecommunications and Information Exchange Between Systems - Protocol Identification in the Network Layer", ISO/IEC TR 9577: 1990 (E) 1990-10-15.

[LANE] "LAN Emulation Over ATM Specification - Version 1.0", ATM Forum af-lane-021.000, January, 1995. 